\title{
LiCl-NaCl-KCl相图的实验测量和热力学优化
}

\author{
卢广轩 ${ }^{1,2}$, 赖添旺 $^{1}$, 何茂刚 ${ }^{1 *}$, 刘向阳 $^{1}$
}

1. 西安交通大学, 热流科学与工程教育部重点实验室, 西安 710049;

2. 西部金属材料股份有限公司, 西安 710201

*联系人, E-mail: mghe@mail.xjtu.edu.cn

2019-08-21 收稿, 2019-09-25 修回, 2019-09-26 接受, 2019-11-22 网络版发表 国家杰出青年科学基金(51525604)资助

\begin{abstract}
摘要 碱金属氯化盐混合物 $\mathrm{LiCl}-\mathrm{NaCl}-\mathrm{KCl}$ 可以作为熔盐电解法制备稀土及其合金的电解质, 该体系的相图是构建 含稀土的多元氯化盐体系热力学数据库的基础. 本文采用差热分析法对二元系 $\mathrm{LiCl}-\mathrm{NaCl}$ 和三元系 $\mathrm{LiCl}-\mathrm{NaCl}-\mathrm{KCl}$ 的相图进行了实验测量, 并基于新的实验数据和文献数据对这两个体系的相图重新进行了热力学优化, 修正了现 有数据库中 $\mathrm{LiCl}-\mathrm{NaCl}-\mathrm{KCl}$ 的热力学参数. 计算得到的 $\mathrm{LiCl}-\mathrm{NaCl}$ 是二元匀晶体系, 极小值点位于 $x_{\mathrm{NaCl}}=0.262$, 温度为 $820 \mathrm{~K}$. 与前人的优化结果相比, 本文计算得到的固相线更接近实验值. 计算结果表明, $\mathrm{LiCl}-\mathrm{NaCl}-\mathrm{KCl}$ 的液相面有一 个极小值点, 而不是四相共晶点, 对应的组分为 $55 \% \mathrm{LiCl}-8 \% \mathrm{NaCl}-37 \% \mathrm{KCl}$, 温度为 $620 \mathrm{~K}$.
\end{abstract}

关键词 LiCl-NaCl-KCl, 修正的似化学模型, 实验测量, 热力学优化

熔盐电解法制备稀土及其合金时，通常使用稀土 氯化盐和碱金属氯化盐组成的混合物作为电解质. 多 元氯化熔盐的物理化学性质对合理选择电解质成分至 关重要. 对熔盐的相平衡性质进行研究, 可以获得电解 质组成和初晶温度的关系, 初步确定电解温度和电解 质成分, 为进一步研究其他性质奠定基础. 工业上最常 用的碱金属氯化盐有 $\mathrm{LiCl}, \mathrm{NaCl}$ 和 $\mathrm{KCl}$, 由这3种氯化盐 组成的混合物体系 $\mathrm{LiCl}-\mathrm{NaCl}-\mathrm{KCl}$ 的相图是构建含稀土 的多元氯化盐体系热力学数据库的基础. 对于二元系 $\mathrm{LiCl}-\mathrm{NaCl}$, 许多研究者使用热分析法或目视变温法对 该体系的相图进行实验测量, 不同研究者得到的该体 系相图差异较大，对该体系是否存在中间化合物及其 是否为二元匀晶体系存在分歧. Sangster和Pelton ${ }^{[1]}$ 对 这些实验研究进行了总结和分析, 并基于这些实验数 据对该体系进行了热力学优化. 他们得到的结论是, 该 体系的相图为具有极小值的二元匀晶相图, $\mathrm{LiCl}$ 与
$\mathrm{NaCl}$ 在高温时可以形成连续固溶体; 随着温度降低, 完 全互溶固溶体转变成两个部分互溶固溶体. 临界点的 计算值为 $514 \mathrm{~K}$, 而实验值多在 $544 \sim 673 \mathrm{~K}$ 之间, 有的实 验值甚至超过 $773 \mathrm{~K}$. 但他们计算得到的固相线与实验 值偏差较大. Tian等人 ${ }^{[2]}$ 对三元系 $\mathrm{LiCl}-\mathrm{NaCl}-\mathrm{LaCl}_{3}$ 的相 图进行实验测量时，使用差热分析法(differential thermal analysis, DTA)和X射线衍射法(X-ray diffraction, $\mathrm{XRD)}$ 对 $\mathrm{LiCl}-\mathrm{NaCl}$ 的相图进行了研究, 证实该体系为二 元匀晶相图. 通过三元系各垂直截面相图的液相线, 可 以得到二元系 $\mathrm{LiCl}-\mathrm{NaCl} 6$ 个组分的初晶温度, 但无法 得到固相线的信息. Sangster和Pelton ${ }^{[1]}$ 使用的是正规溶 液模型. 后来Chartrand和Pelton ${ }^{[3]}$ 将其参数转换为修正 的似化学模型参数, 得到了相同的相图. 鉴于该体系固 相线的计算值与实验值偏差较大，且目前的实验数据 多为1910 1960年采用步冷曲线法测得的，一致性较 差, 本文采用DTA方法对该体系的相图进行重新测量.

引用格式: 卢广轩, 赖添旺, 何茂刚, 等. LiCl-NaCl-KCl相图的实验测量和热力学优化. 科学通报, 2020, 65: 641-648 Lu G X, Lai T W, He M G, et al. Experimental measurement and thermodynamic optimization of the phase diagram of LiCl-NaCl-KCl system (in Chinese). Chin Sci Bull, 2020, 65: 641-648, doi: 10.1360/TB-2019-0416 
对于三元系 LiCl-NaCl-KCl, Sangser和Pelton ${ }^{[4]}$ 也对 相关实验研究进行了总结, 并对该体系进行了热力学 优化. 他们得到的相图含有一个四相共晶点, 共晶温度 的计算值为 $619 \mathrm{~K}$, 在实验值610 630 K范围之内, 共晶 组分为 $55 \% \mathrm{LiCl}-9 \% \mathrm{NaCl}-36 \% \mathrm{KCl}$. 但是根据FactSage 软件中的FTsalt数据库计算得到的相图没有四相共晶 点, 只有一个极小值点, 温度为 $621 \mathrm{~K}$, 组分为 $55 \% \mathrm{LiCl}-$ $7 \% \mathrm{NaCl}-38 \% \mathrm{KCl}$. 鉴于此, 本文对该组分附近的局部相 图进行了实验测量, 然后基于所测得的二元系 LiCl$\mathrm{NaCl}$ 和三元系 $\mathrm{LiCl}-\mathrm{NaCl}-\mathrm{KCl}$ 的实验数据, 结合文献数 据, 对其进行了热力学优化.

\section{LiCl-NaCl和LiCl-NaCl-KCl相图的 实验测量}

\section{1 相图的实验测量}

本实验使用的纯质 $\mathrm{LiCl}, \mathrm{NaCl}$ 和 $\mathrm{KCl}$ 购自上海阿拉 丁生化科技股份有限公司，纯度均为 $99.99 \%$. 纯质 $\mathrm{LiCl}$ 和 $\mathrm{KCl}$ 在空气中容易潮解, 在制备样品前, 先将其置于 真空干燥箱内, 温度保持 $200^{\circ} \mathrm{C}$ 加热 $2 \mathrm{~h}$. 对相图的实验 测量采用的是北京京仪高科仪器有限公司的差热分析 仪, 仪器型号为ZCR-B, 测温量程为室温 $1450^{\circ} \mathrm{C}$. 热分 析时使用的坩埚为铂金坩埚, 利用标准物质 $\mathrm{In}, \mathrm{SiO}_{2}$ 和 $\mathrm{SrCO}_{3}$ 进行温度校准, 温度的最大偏差为 $2 \mathrm{~K}$. 对每个样 品采集加热和冷却DTA曲线, 以分析相变温度. 所有的 测量均在高纯氮气 $(99.99 \%)$ 保护气氛下进行, 气流速度 为 $50 \mathrm{~mL} \mathrm{~min}^{-1}$, 试样以 $5 \sim 10 \mathrm{~K} \mathrm{~min}^{-1}$ 的速率加热和冷却 各两次. 首先测量纯质 $\mathrm{LiCl}, \mathrm{NaCl}$ 和 $\mathrm{KCl}$ 的熔点, 然后测 量5个不同组分的 $\mathrm{LiCl}-\mathrm{NaCl}$ 混合物, 最后测量 5 个不同 组分的 LiCl-NaCl-KCl混合物，对每个组分进行3次测 量, 求取平均值. 所有样品都在干燥的有氮气保护的手 套箱中按所需比例称量, 然后在玛瑙研钵内研磨, 并尽 量使其混合均匀. 为了避免过冷效应以及使样品混合 均匀, 本实验主要对第二次加热曲线的热效应进行 分析.

\section{2 实验测量结果}

首先对纯质样品的熔点进行测量, 以检验样品纯 度及仪器对此类物质相变温度的测量精度. 图1给出了 纯质 $\mathrm{LiCl}, \mathrm{NaCl}$ 和 $\mathrm{KCl}$ 的DTA曲线, 从曲线上分析得到 的它们的熔点分别为 $881.9,1074.0$ 和 $1046.0 \mathrm{~K}$. Fact$\mathrm{Sage}$ 数据库中评估过的它们的熔点分别为 883.0 ,

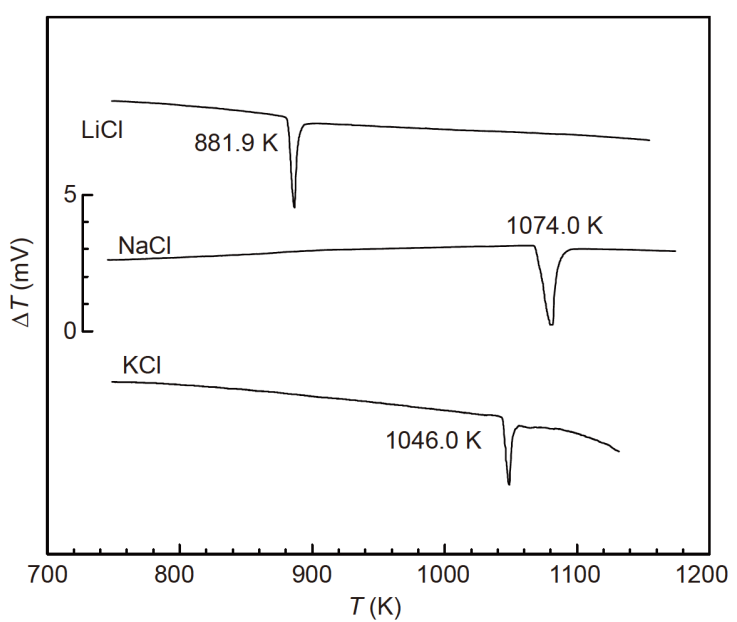

图 1 纯质 $\mathrm{LiCl}, \mathrm{NaCl}$ 和KCl的DTA曲线

Figure 1 DTA curves of pure $\mathrm{LiCl}, \mathrm{NaCl}$ and $\mathrm{KCl}$

1073.8 和 $1044.0 \mathrm{~K}$, 本文实验测量值与之吻合良好.

$\mathrm{LiCl}-\mathrm{NaCl}$ 相图的研究主要是根据 5 个不同组分样 品的DTA数据进行分析. 实验测得的这 5 个组分的固相 线和液相线温度如表1所示, 图2给出了它们的DTA加 热曲线. 从图 2 可以看出, 除第一条曲线外, 其余曲线都 有两个峰. 根据前人的实验测量结果 ${ }^{[1]}$ 和FactSage软件 的数据库计算结果可知, 这两个峰分别对应相图的固 相线和液相线. 因此, 第一个峰的外推起始点用来确定 固相线温度，第二个峰的峰值点用来确定液相线温 度 $^{[5]}$. 第一条曲线只有一个峰的原因是该组分在极小 值点附近, 固相线和液相线的温度很接近, 两个峰发生 重叠, 难以区分开.

根据目前已知的实验数据以及Sangser和Pelton ${ }^{[4]}$ 的优化结果, $\mathrm{LiCl}-\mathrm{NaCl}-\mathrm{KCl}$ 的共晶点组分在 $55 \% \mathrm{LiCl}-$ $9 \% \mathrm{NaCl}-36 \% \mathrm{KCl}$ 附近, 因此本文选取该体系的 $x_{\mathrm{NaCl}}=$ 0.09 和 $x_{\mathrm{LiCl}}=0.55$ 两个截面进行测量. 每个截面都测量了 两个不同组分, 还测量了这两个截面的交点组分, 得到 了 5 个不同组分的DTA加热曲线. 实验测得的这 5 个组

表 1 二元系 LiCl-NaCl的DTA测量结果

Table 1 Experimental data from DTA curves of the $\mathrm{LiCl}-\mathrm{NaCl}$ system

\begin{tabular}{ccc}
\hline 混合物组分 & 固相线温度 $(\mathrm{K})$ & 液相线温度 $(\mathrm{K})$ \\
\hline $71 \% \mathrm{LiCl}-29 \% \mathrm{NaCl}$ & 821.8 & \\
$59 \% \mathrm{LiCl}-41 \% \mathrm{NaCl}$ & 827.1 & 877.5 \\
$48 \% \mathrm{LiCl}-52 \% \mathrm{NaCl}$ & 834.3 & 922.1 \\
$40 \% \mathrm{LiCl}-60 \% \mathrm{NaCl}$ & 842.3 & 944.6 \\
$24 \% \mathrm{LiCl}-76 \% \mathrm{NaCl}$ & 889.2 & 996.0 \\
\hline
\end{tabular}




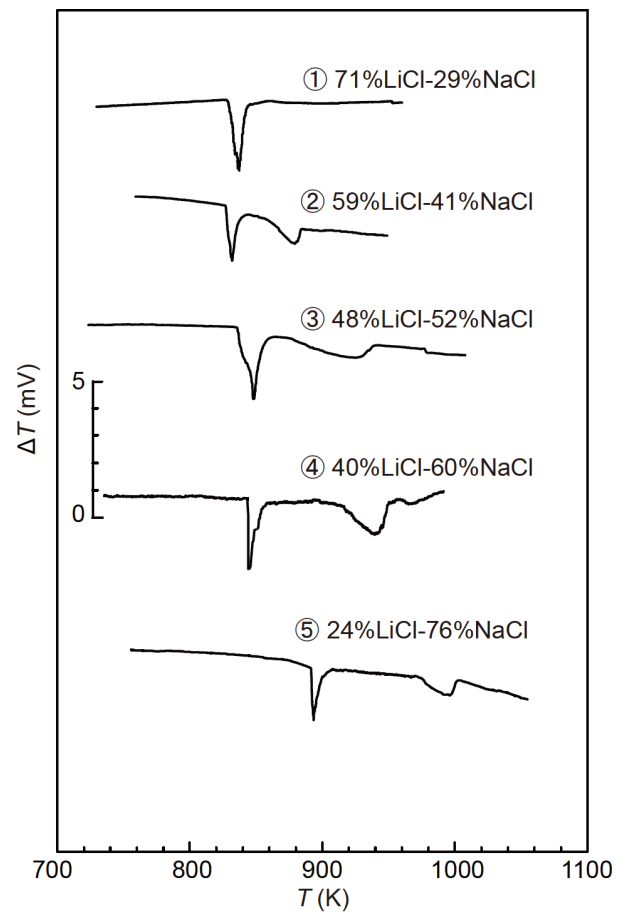

图 $2 \mathrm{LiCl}-\mathrm{NaCl}$ 混合物的DTA曲线

Figure 2 DTA curves of $\mathrm{LiCl}-\mathrm{NaCl}$ mixtures

分的固相线和液相线温度如表2所示，图3给出了它们 的DTA曲线. 除曲线(3)外, 其他曲线都有两个峰. 同样 地, DTA曲线上的这两个峰应分别对应固相线和液相 线温度. 曲线(3)只有一个峰的原因是该点靠近共晶点, 液相线温度与固相线温度非常接近.

\section{LiCl-NaCl-KCl体系的热力学优化}

\section{1 纯质的热力学性质}

Chartrand和Pelton ${ }^{[3]}$ 已经根据Barin ${ }^{[6]}$ 编辑的无机物 热力学数据集对纯质 $\mathrm{LiCl}, \mathrm{NaCl}$ 和 $\mathrm{KCl}$ 的热力学性质 $\left(\Delta H_{298.15 \mathrm{~K}}^{0}, S_{298.15 \mathrm{~K}}^{0}\right.$ 和 $\left.C_{p}\right)$ 进行过评估, 可以直接从FactSage软件的FTsalt数据库中获取这些数据, 如表3所示.

\section{2 热力学模型}

采用修正的似化学模型 ${ }^{[7,8]}$ 描述该体系液相. 假设 离子溶液中存在阴阳离子两个亚点阵．对于只含有一 种阴离子的二元系 $\mathrm{AX}-\mathrm{BX}$ ，阳离子 $\mathrm{A}$ 和 $\mathrm{B}$ 位于阳离子亚 点阵，阴离子 $\mathrm{X}$ 位于阴离子亚点阵. 在溶液中会发生第 二最近邻对交换反应 $[A-A]+[B-B]=2[A-B]$, 该反应生成 2 mol[A-B]对的吉布斯(Gibbs)自由能变化量为 $\Delta g_{\mathrm{AB}}$.
表 2 三元系 LiCl-NaCl-KCl的DTA测量结果

Table 2 Experimental data from DTA curves of the LiCl-NaCl-KCl system

\begin{tabular}{ccc}
\hline 混合物组分 & 固相线温度 $(\mathrm{K})$ & 液相线温度 $(\mathrm{K})$ \\
\hline $66 \% \mathrm{LiCl}-9 \% \mathrm{NaCl}-25 \% \mathrm{KCl}$ & 622.9 & 707.2 \\
$43 \% \mathrm{LiCl}-9 \% \mathrm{NaCl}-48 \% \mathrm{KCl}$ & 622.5 & 753.5 \\
$55 \% \mathrm{LiCl}-9 \% \mathrm{NaCl}-36 \% \mathrm{KCl}$ & 621.3 & \\
$55 \% \mathrm{LiCl}-27 \% \mathrm{NaCl}-18 \% \mathrm{KCl}$ & 621.7 & 788.7 \\
$55 \% \mathrm{LiCl}-18 \% \mathrm{NaCl}-27 \% \mathrm{KCl}$ & 620.9 & 713.0 \\
\hline
\end{tabular}

二元系AX-BX的液相溶液的吉布斯自由能表达 式为

$G=n_{\mathrm{AX}} g_{\mathrm{AX}}^{0}+n_{\mathrm{BX}} g_{\mathrm{BX}}^{0}-T \Delta S^{\text {config }}+n_{\mathrm{AB}}\left(\Delta g_{\mathrm{AB}} / 2\right)$,

式中 $n_{\mathrm{AX}}$ 和 $n_{\mathrm{BX}}$ 分别是纯质 $\mathrm{AX}$ 和 $\mathrm{BX}$ 的摩尔数, $g_{\mathrm{AX}}^{0}$ 和 $g_{\mathrm{BX}}^{0}$ 分别是纯质 $\mathrm{AX}$ 和 $\mathrm{BX}$ 的摩尔吉布斯自由能, $n_{\mathrm{AB}}$ 是 $[\mathrm{A}-\mathrm{B}$ ] 对的摩尔数. 假设 $[\mathrm{A}-\mathrm{A}],[\mathrm{B}-\mathrm{B}]$ 和 $[\mathrm{A}-\mathrm{B}]$ 对在溶液中随机 分布，可以得到正好符合一维伊辛模型(Ising model)的 $\Delta S^{\text {config }}$ 表达式:

$$
\begin{aligned}
\Delta S^{\text {config }}= & -R\left(n_{\mathrm{A}} \ln X_{\mathrm{A}}+n_{\mathrm{B}} \ln X_{\mathrm{B}}\right) \\
& -R\left[n_{\mathrm{AA}}\left(\frac{X_{\mathrm{AA}}}{Y_{\mathrm{A}}^{2}}\right)+n_{\mathrm{BB}}\left(\frac{X_{\mathrm{BB}}}{Y_{\mathrm{B}}^{2}}\right)+n_{\mathrm{AB}}\left(\frac{X_{\mathrm{AB}}}{2 Y_{\mathrm{A}} Y_{\mathrm{B}}}\right)\right],
\end{aligned}
$$

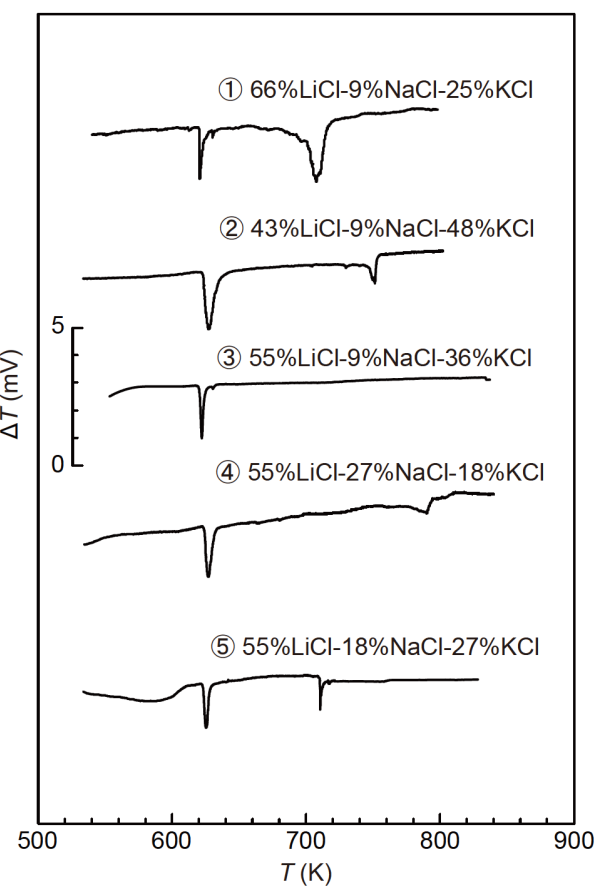

图 3 LiCl-NaCl-KCl混合物的DTA曲线

Figure 3 DTA curves of LiCl-NaCl-KCl mixtures 
表 3 纯质 $\mathrm{LiCl}, \mathrm{NaCl}$ 和 $\mathrm{KCl}$ 的热力学性质 ${ }^{\mathrm{a}}$

Table 3 Thermodynamic properties of pure $\mathrm{LiCl}, \mathrm{NaCl}$ and $\mathrm{KCl}$

\begin{tabular}{cccccc}
\hline & & $T(\mathrm{~K})$ & $\Lambda H_{298.15 \mathrm{~K}}^{0}\left(\mathrm{~J} \mathrm{~mol}^{-1}\right)$ & $S_{298.15 \mathrm{~K}}^{0}\left(\mathrm{~J} \mathrm{~K}^{-1} \mathrm{~mol}^{-1}\right)$ & $C_{p}\left(\mathrm{~J} \mathrm{~K}^{-1} \mathrm{~mol}^{-1}\right)$ \\
\hline \multirow{2}{*}{$\mathrm{LiCl}$} & $\mathrm{S}$ & $298.15 \sim 2000$ & -408266.4 & 59.2998 & $41.4174+0.0233969 T$ \\
& $\mathrm{~L}$ & $298.15 \sim 2000$ & -395776.3 & 66.2774 & $73.3832-0.0094726 T$ \\
$\mathrm{NaCl}$ & $\mathrm{S}$ & $298.15 \sim 2000$ & -411119.8 & 72.1322 & $45.9403+0.0163176 T$ \\
& $\mathrm{~L}$ & $298.15 \sim 1500$ & -394956.0 & 76.0761 & $77.7638+0.0075312 T$ \\
& $\mathrm{~L}$ & $1500 \sim 2000$ & & & 66.9440 \\
$\mathrm{KCl}$ & $\mathrm{S}$ & $298.15 \sim 2500$ & -436684.1 & 82.5503 & $40.0158+0.0254680 T+364846 T^{-2}$ \\
& $\mathrm{~L}$ & $298.15 \sim 2500$ & -421824.9 & 86.5225 & 73.5966 \\
\hline
\end{tabular}

a) S表示固相, L表示液相

式中 $R$ 为通用气体常数, $n_{i}$ 和 $X_{i}$ 分别是阳离子亚点阵中 组元 $i(i=\mathrm{A}$ 或 $\mathrm{B})$ 的摩尔数和摩尔分数, $Y_{i}$ 是组元 $i$ 的配位 数当量摩尔分数, $n_{i j}$ 和 $X_{i j}$ 分别是 $[i-j]$ 对的摩尔数和摩尔 分数. $X_{i}, Y_{i}$ 和 $X_{i j}$ 的表达式分别为

$$
\begin{aligned}
X_{i} & =n_{i} /\left(n_{\mathrm{A}}+n_{\mathrm{B}}\right), \\
Y_{i} & =Z_{i} n_{i} /\left(Z_{\mathrm{A}} n_{\mathrm{A}}+Z_{\mathrm{B}} n_{\mathrm{B}}\right) \\
& =Z_{i} X_{i} /\left(Z_{\mathrm{A}} X_{\mathrm{A}}+Z_{\mathrm{B}} X_{\mathrm{B}}\right), \\
X_{i j} & =n_{i j} /\left(n_{\mathrm{AA}}+n_{\mathrm{BB}}+n_{\mathrm{AB}}\right),
\end{aligned}
$$

式中 $Z_{\mathrm{A}}$ 和 $Z_{\mathrm{B}}$ 分别是阳离子 $\mathrm{A}$ 和 $\mathrm{B}$ 的配位数, 它们与 $n_{i}$ 和 $n_{i j}$ 的关系为

$$
\begin{aligned}
& Z_{\mathrm{A}} n_{\mathrm{A}}=2 n_{\mathrm{AA}}+n_{\mathrm{AB}}, \\
& Z_{\mathrm{B}} n_{\mathrm{B}}=2 n_{\mathrm{BB}}+n_{\mathrm{AB}} .
\end{aligned}
$$

将式(5) (7)代人到式(4)中, 可以得到:

$Y_{\mathrm{A}}=X_{\mathrm{AA}}+X_{\mathrm{AB}} / 2, Y_{\mathrm{B}}=X_{\mathrm{BB}}+X_{\mathrm{AB}} / 2$.

随溶液成分变化的配位数 $Z_{\mathrm{A}}$ 和 $Z_{\mathrm{B}}$ 的表达式为

$\frac{1}{Z_{\mathrm{A}}}=\frac{1}{Z_{\mathrm{AA}}^{\mathrm{A}}}\left(\frac{2 n_{\mathrm{AA}}}{2 n_{\mathrm{AA}}+n_{\mathrm{AB}}}\right)+\frac{1}{Z_{\mathrm{AB}}^{\mathrm{A}}}\left(\frac{n_{\mathrm{AB}}}{2 n_{\mathrm{AA}}+n_{\mathrm{AB}}}\right)$,

$\frac{1}{Z_{\mathrm{B}}}=\frac{1}{Z_{\mathrm{BB}}^{\mathrm{B}}}\left(\frac{2 n_{\mathrm{BB}}}{2 n_{\mathrm{BB}}+n_{\mathrm{AB}}}\right)+\frac{1}{Z_{\mathrm{AB}}^{\mathrm{B}}}\left(\frac{n_{\mathrm{AB}}}{2 n_{\mathrm{BB}}+n_{\mathrm{AB}}}\right)$.

将式(9)和(10)代人到式(6)和式(7)中, 可以得到两 个较为简单的表达式:

$n_{\mathrm{A}}=2 n_{\mathrm{AA}} / Z_{\mathrm{AA}}^{\mathrm{A}}+n_{\mathrm{AB}} / Z_{\mathrm{AB}}^{\mathrm{A}}$,

$n_{\mathrm{B}}=2 n_{\mathrm{BB}} / Z_{\mathrm{BB}}^{\mathrm{B}}+n_{\mathrm{AB}} / Z_{\mathrm{BA}}^{\mathrm{B}}$,

式中 $Z_{\mathrm{AA}}^{\mathrm{A}}$ 是当组元 $\mathrm{A}$ 的最近邻都是 $\mathrm{A}$ 时的配位数, $Z_{\mathrm{AB}}^{\mathrm{A}}$ 是 当组元 $\mathrm{A}$ 的最近邻都是 $\mathrm{B}$ 时的配位数, $Z_{\mathrm{BB}}^{\mathrm{B}}$ 和 $Z_{\mathrm{BA}}^{\mathrm{B}}$ 分别是 当组元 $B$ 的最近邻都是 $B$ 或者都是 $A$ 时的配位数.
需要说明的是, 由于没有精确的构型熵 $\Delta S^{\mathrm{config}}$ 三维 表达式, 式(2)仅是对三维形式的近似. 所以，为了获得 与实验数据更吻合的计算结果，可以通过选择合适的 配位数来补偿混合构型熵近似表达式与实际值的偏差. 因此，该模型的配位数未必是亚点阵中各组元的真实 配位数.

当体系处于平衡态时, 其总吉布斯自由能最小. 平 衡时溶液中的对分布应该满足

$\left(\frac{\partial G}{\partial n_{\mathrm{AB}}}\right)_{n_{\mathrm{A}}, n_{\mathrm{B}}}=0$.

可以推导出式(14)所示的“似化学反应”的“平衡 常数”:

$\frac{X_{\mathrm{AB}}^{2}}{X_{\mathrm{AA}} X_{\mathrm{AB}}}=4 \exp \left(\frac{-\Delta g_{\mathrm{AB}}}{R T}\right)$.

$\Delta g_{\mathrm{AB}}$ 可以表示为 $[\mathrm{A}-\mathrm{A}]$ 和 $[\mathrm{B}-\mathrm{B}]$ 对的摩尔分数的 函数:

$\Delta g_{\mathrm{AB}}=\Delta g_{\mathrm{AB}}^{0}+\sum_{i+j \geq 1} g_{\mathrm{AB}}^{i j} X_{\mathrm{AA}}^{i} X_{\mathrm{BB}}^{i}$,

式中 $\Delta g_{\mathrm{AB}}^{0}$ 和 $g_{\mathrm{AB}}^{i j}$ 均为模型参数.

本文所选择的第二最近邻对配位数与Chartrand和 Pelton ${ }^{[3]}$ 的选择保持一致:

$Z_{\mathrm{LiLi}}^{\mathrm{Li}}=Z_{\mathrm{NaNa}}^{\mathrm{Na}}=Z_{\mathrm{KK}}^{\mathrm{K}}=Z_{\mathrm{LiNa}}^{\mathrm{Na}}=Z_{\mathrm{LiNa}}^{\mathrm{Li}}$

$=Z_{\mathrm{NaK}}^{\mathrm{Na}}=Z_{\mathrm{NaK}}^{\mathrm{K}}=Z_{\mathrm{LiK}}^{\mathrm{Li}}=Z_{\mathrm{LiK}}^{\mathrm{K}}=6$.

对二元体系进行热力学优化的过程就是确定该二 元系吉布斯自由能的过程，而三元系的吉布斯自由能 可以通过几何模型由各二元系的热力学参数得到, 进 而可以对该三元系的相图等热力学性质进行预测，再 根据三元系的实验数据决定是否需要添加三元热力学 
参数. 由于 $\mathrm{LiCl}, \mathrm{NaCl}$ 和 $\mathrm{KCl}$ 性质相似, 本文选择的几何 模型为对称模型—Kohler模型，这也与Chartrand和 Pelton ${ }^{[3]}$ 的选择一致. 对于 $\mathrm{LiCl}$ 和 $\mathrm{NaCl}$ 形成的固溶体, 使用化合物能量模型 ${ }^{[9,10]}$ 来描述.

\section{3 热力学优化结果及分析}

首先对二元系 $\mathrm{LiCl}-\mathrm{NaCl}$ 重新进行热力学优化，使 用的实验数据包括文献中的相图数据、固液混合焓数 据以及本文测得的相图数据. 然后结合FactSage软件中 FTsalt数据库中的 $\mathrm{LiCl}-\mathrm{KCl}$ 和 $\mathrm{NaCl}-\mathrm{KCl}$ 的优化结果, 使 用本文的实验数据对 $\mathrm{LiCl}-\mathrm{NaCl}-\mathrm{KCl}$ 的相图进行优化.

Klochko ${ }^{[11]}$ 分别使用目视变温法和热分析法测量 了 $\mathrm{LiCl}-\mathrm{NaCl}$ 的液相线和固相线, Gromakov和Gromako$\mathrm{va}^{[12]}$ 使用热分析法对该体系相图进行了测量, 而Tian等 人 $^{[2]}$ 使用差热分析法对液相线进行了研究. 在富 $\mathrm{LiCl}$ 侧, 他们测得的固相线和液相线基本一致; 而在富 $\mathrm{NaCl}$ 侧, Klochko ${ }^{[11]}$ 与 Tian等人 ${ }^{[2]}$ 测得的液相线比Gromakov 和Gromakova ${ }^{[12]}$ 测得的略高, 而Klochko ${ }^{[11]}$ 测得的固相 线比Gromakov和Gromakova ${ }^{[12]}$ 测得的高很多, 有的组 分处甚至相差 30 K. Sangster和Pelton ${ }^{[1]}$ 优化计算得到的 液相线接近Klochko ${ }^{[11]}$ 与 Tian等人 ${ }^{[2]}$ 测得的液相线, 计 算得到的固相线比Klochko ${ }^{[11]}$ 的测量结果低很多，与 Gromakov和Gromakova ${ }^{[12]}$ 的测量值接近. 可以看出, 该 体系的固相线有比较大的争议. 本文对该体系相图进 行测量后发现, 得到的固相线与Gromakov和Gromako$\mathrm{va}^{[12]}$ 的测量结果较接近. 除此之外, Klochko ${ }^{[1]}$ 测得的 极小值点的组分为 $x_{\mathrm{NaCl}}=0.29$, 温度为 $801 \mathrm{~K}$; Gromakov 和Gromakova ${ }^{[12]}$ 测得的极小值点的组分为 $x_{\mathrm{NaCl}}=0.275$, 温度为 $819 \mathrm{~K} ;$ Sangster和Pelton ${ }^{[1]}$ 充分考虑多组实验数 据后优化得到的极小值点位于 $x_{\mathrm{NaCl}}=0.28$, 温度为 $827 \mathrm{~K}$. 本文利用修正的似化学模型对该体系重新进行 优化, 考虑了上述所有相图数据, 并给予测量得到的相 图数据较大权重. Hersh和Kleppa ${ }^{[13]}$ 还使用高温反应量 热法测量了固相 $\mathrm{NaCl}$ 和液相 $\mathrm{LiCl}$ 在 $1013 \mathrm{~K}$ 时的混合焓 数据, 本文在优化时一并考虑.

优化得到的 $\Delta g_{\mathrm{AB}}$ 为

$\Delta g_{\mathrm{LiNa} / \mathrm{Cl}}=-1562.0+0.2000 T$.

对于 $\mathrm{LiCl}$ 和 $\mathrm{NaCl}$ 形成的固溶体，所使用的化合物 能量模型为 $(\mathrm{Li}, \mathrm{Na}) \mathrm{Cl}$, 其过量吉布斯自由能为

$G_{\mathrm{LiNa}}^{\mathrm{E}}=7500.0 y_{\mathrm{Li}} y_{\mathrm{Na}}+4600.0 y_{\mathrm{Li}} y_{\mathrm{Na}}\left(y_{\mathrm{Li}}-y_{\mathrm{Na}}\right)$.
优化得到的 $\mathrm{LiCl}-\mathrm{NaCl}$ 相图和 $1013 \mathrm{~K}$ 时的固相 $\mathrm{NaCl}$ 和液相 $\mathrm{LiCl}$ 混合焓与实验值的对比分别如图 4 和 5 所示. 可以看出, 计算值与本文实验值吻合得很好. 优 化得到的匀晶相图的极小值点组分为 $x_{\mathrm{NaCl}}=0.262$, 温度 为 $820 \mathrm{~K}$, 与 Gromakov和Gromakova ${ }^{[12]}$ 的测量结果接近. 而本文测得的 $71 \% \mathrm{LiCl}-29 \% \mathrm{NaCl}$ 组分也确实在极小值 点附近. 图6给出了计算得到的该体系在300 1100 K的 完整相图. 对于液相线, 本文模型与Sangster和Pelton ${ }^{[1]}$ 的模型计算结果相近，计算值与实验值相对偏差的绝 对平均值分别为 $0.5 \%$ 和 $0.6 \%$, 最大偏差均不超过 $10 \mathrm{~K}$; 对于固相线，相对偏差的绝对平均值分别为 $0.8 \%$ 和 $1.9 \%$. 需要指出的是, Sangster和Pelton ${ }^{[1]}$ 计算得到的固 相线与实验值的最大偏差约为 $30 \mathrm{~K}$, 而本文模型的最 大偏差为 $15 \mathrm{~K}$. 从图6可以看出，本文计算得到的该体

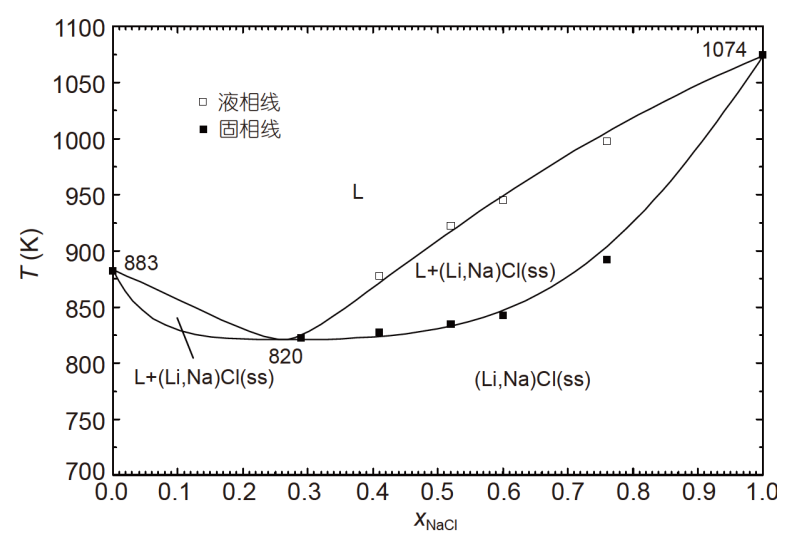

图 $4 \mathrm{LiCl}-\mathrm{NaCl}$ 体系相图计算值与本文实验值的对比

Figure 4 Comparison of the calculated phase diagram of $\mathrm{LiCl}-\mathrm{NaCl}$ system and experimental data measured by this work

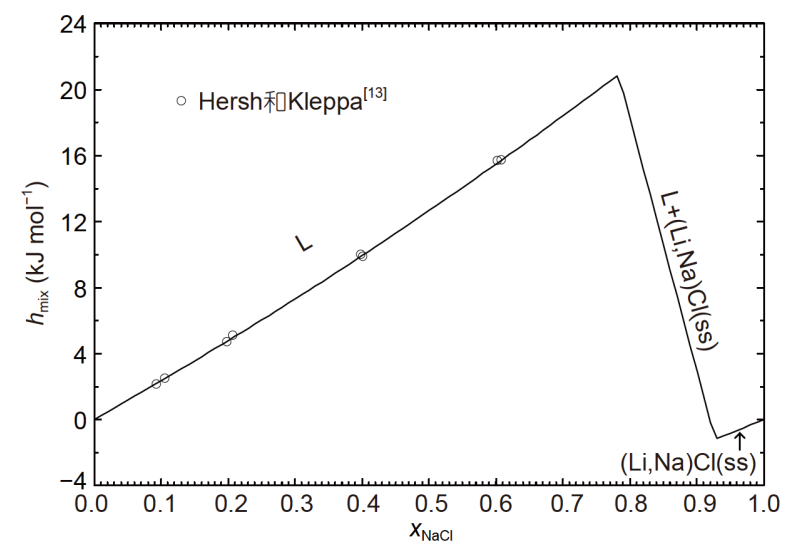

图 $51013 \mathrm{~K}$ 时 $\mathrm{LiCl}-\mathrm{NaCl}$ 体系中固相 $\mathrm{NaCl}$ 与液相 $\mathrm{LiCl}$ 的混合焓 Figure 5 The mixing enthalpy of solid $\mathrm{NaCl}$ and liquid $\mathrm{LiCl}$ in $\mathrm{LiCl}-$ $\mathrm{NaCl}$ system at $1013 \mathrm{~K}$ 


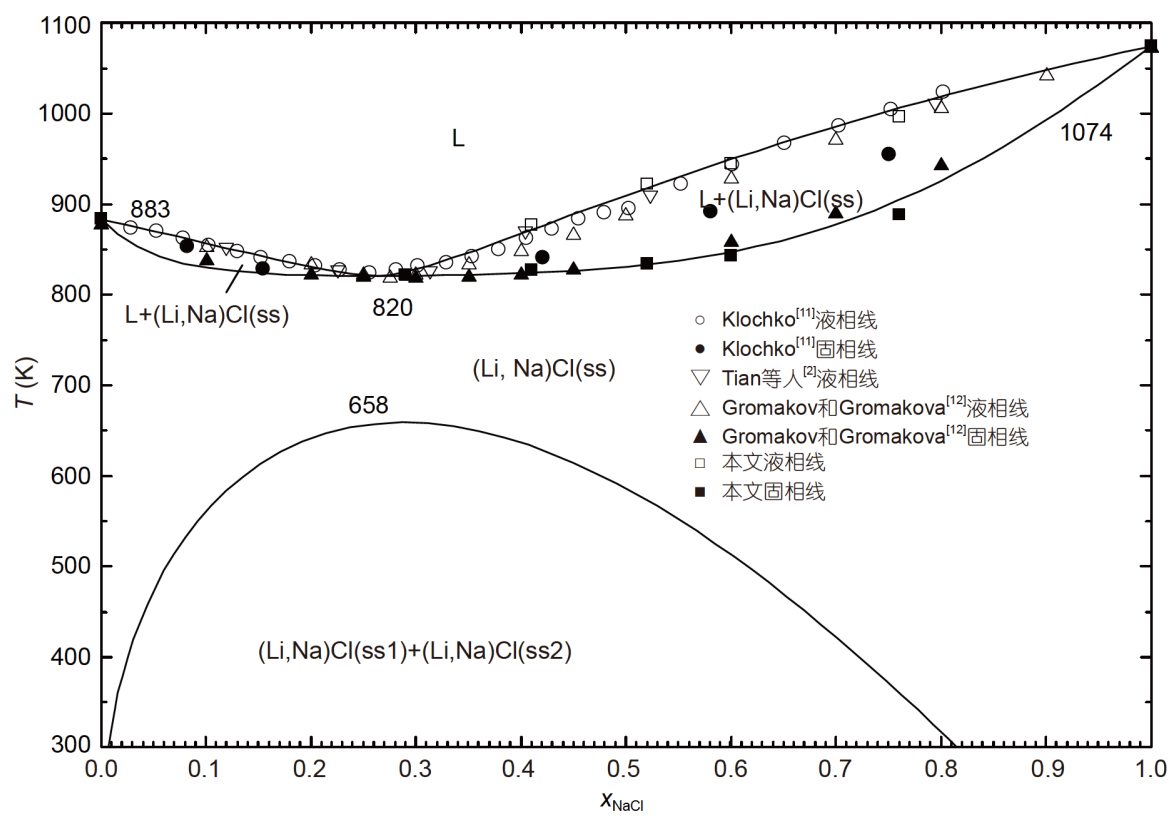

图 $6 \mathrm{LiCl}-\mathrm{NaCl}$ 体系的完整相图

Figure 6 The complete phase diagram of $\mathrm{LiCl}-\mathrm{NaCl}$ system

系在低温下出现固溶体相分离的临界点温度为 $658 \mathrm{~K}$, 组分为 $x_{\mathrm{NaCl}}=0.293$. 临界点温度的实验值差异较大, 多 在544 673 K之间, 本文计算值在该范围内, 而Sangster 和Pelton ${ }^{[1]}$ 计算得到的临界点温度为 $514 \mathrm{~K}$, 超出了实验 值范围. 可以看出, 与Sangster和Pelton ${ }^{[1]}$ 的计算结果相 比, 本文计算得到的 $\mathrm{LiCl}-\mathrm{NaCl}$ 相图的固相线和固溶体 发生分离的临界点与实验值更吻合, 因此所得到的模 型参数更合理.

在优化三元系 LiCl-NaCl-KCl时, $\mathrm{LiCl}-\mathrm{KCl}$ 和 NaCl-

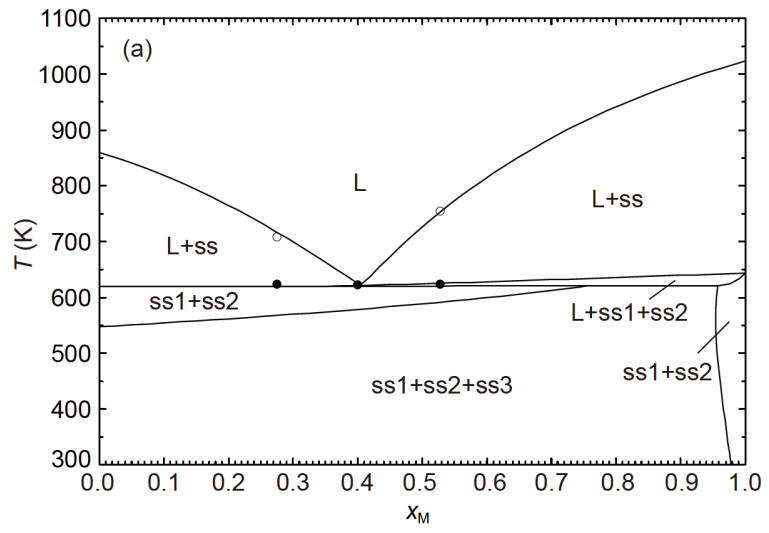

$\mathrm{KCl}$ 的热力学参数以及固溶体的处理方式与文献[3]保 持一致. 与 $\mathrm{LiCl}-\mathrm{NaCl}$ 体系类似, 固相 $\mathrm{NaCl}$ 与 $\mathrm{KCl}$ 也可以 形成无限互溶固溶体. 而 $\mathrm{LiCl}$ 与 $\mathrm{KCl}$ 之间的互溶度很小, 在本文计算中忽略不计, 将二元系 $\mathrm{LiCl}-\mathrm{KCl}$ 当作简单低 共熔体系来处理. 在三元系中, $\mathrm{LiCl}, \mathrm{NaCl}$ 和 $\mathrm{KCl}$ 可以一 起形成固溶体, 该固溶体的吉布斯自由能由化合物能 量模型 $(\mathrm{Li}, \mathrm{Na}, \mathrm{K}) \mathrm{Cl}$ 来描述. 为了保证计算得到的二元 系 $\mathrm{LiCl}-\mathrm{KCl}$ 为低共熔体系, 将固溶体模型中该体系的过 量吉布斯自由能设置为

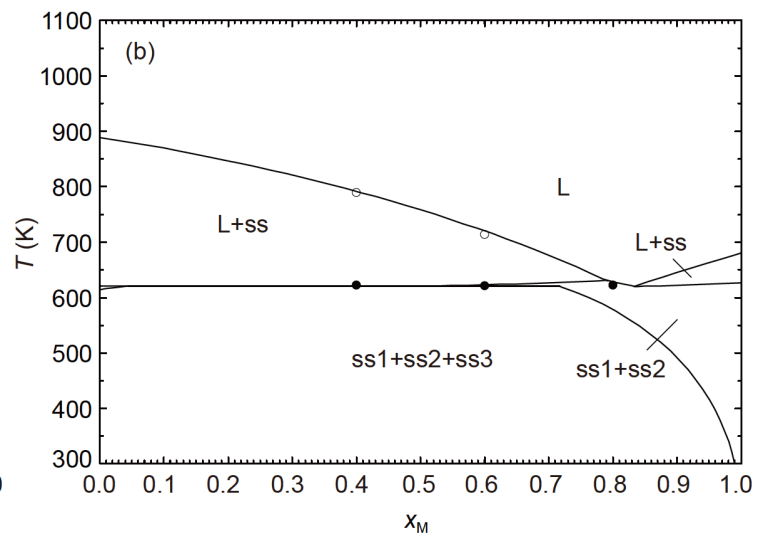

图 $7 \mathrm{LiCl}-\mathrm{NaCl}-\mathrm{KCl}$ 体系在截面 $x_{\mathrm{NaCl}}=0.09$ 和 $x_{\mathrm{LiCl}}=0.55$ 的相图. (a) $x_{\mathrm{NaCl}}=0.09, \mathrm{M}=(\mathrm{KCl})_{0.91}(\mathrm{NaCl})_{0.09} ;$ (b) $x_{\mathrm{LiCl}}=0.55, \mathrm{M}=(\mathrm{KCl})_{0.45}(\mathrm{LiCl})_{0.55} . \mathrm{ss}, \mathrm{ss} 1$, $\mathrm{ss} 2$ 和 $\mathrm{ss} 3$ 分别为 $\mathrm{LiCl}, \mathrm{NaCl}$ 和 $\mathrm{KCl}$ 形成的固溶体

Figure 7 Vertical section phase diagram of the LiCl-NaCl- $\mathrm{KCl}$ system at $x_{\mathrm{NaCl}}=0.09$ and $x_{\mathrm{LiCl}}=0.55$. (a) $x_{\mathrm{NaCl}}=0.09, \mathrm{M}=(\mathrm{KCl})_{0.91}(\mathrm{NaCl})_{0.09} ;(\mathrm{b})$ $x_{\mathrm{LiCl}}=0.55, \mathrm{M}=(\mathrm{KCl})_{0.45}(\mathrm{LiCl})_{0.55}$. ss, ss1, ss2 and ss3 are the sosoloid formed by $\mathrm{LiCl}, \mathrm{NaCl}$ and $\mathrm{KCl}$, respectively 
$G_{\mathrm{LiK}}^{\mathrm{E}}=100000 y_{\mathrm{Li}} y_{\mathrm{K}}$.

由于无法获取其他三元系实验数据，本文在对该 体系进行优化时只考虑本文测得的实验数据及文献[4] 中的四相共晶点数据. 为了使计算值与实验值相吻合, 需要添加一个三元参数:

$\Delta g_{\mathrm{KNa}(\mathrm{Li})}^{001}=-2900.0$.

图7给出了截面 $x_{\mathrm{NaCl}}=0.09$ 和 $x_{\mathrm{LiCl}}=0.55$ 的垂直截面 相图的计算值与本文实验值的对比. 可以看出, 计算值 与实验值吻合得很好. 图8给出了三元系 $\mathrm{LiCl}-\mathrm{NaCl}-\mathrm{KCl}$ 的液相面投影图，在图中也标出了本文实验测量的 5 个 不同组分的位置. 可以看出, 本文计算的该相图液相面 含有一个极小值点 $\mathrm{M}$, 温度为 $620 \mathrm{~K}$, 对应的组分为 $55 \%$ $\mathrm{LiCl}-8 \% \mathrm{NaCl}-37 \% \mathrm{KCl}$, 与文献值(共晶温度 $619 \mathrm{~K}$, 组分 $55 \% \mathrm{LiCl}-9 \mathrm{NaCl} \%-36 \% \mathrm{KCl})^{[4]}$ 十分接近. 需要指出的是, 尽管前人在测量 $\mathrm{LiCl}-\mathrm{NaCl}-\mathrm{KCl}$ 的相图时认为该点为四 相共晶点，但在实验中并没有对共晶点附近的相结构 进行分析，无法确认在该点处有 3 种固相. 根据本文的 计算结果, 如图7所示, 该点处只有两种固溶体相, 而且 是由一种固溶体相分离而来, 这也与用FactSage软件中 的数据库计算得到的结果相一致.

\section{3 结论}

采用DTA方法对二元系 LiCl-NaCl和三元系 LiCl$\mathrm{NaCl}-\mathrm{KCl}$ 的相图进行了测量, 并对其相图重新进行了

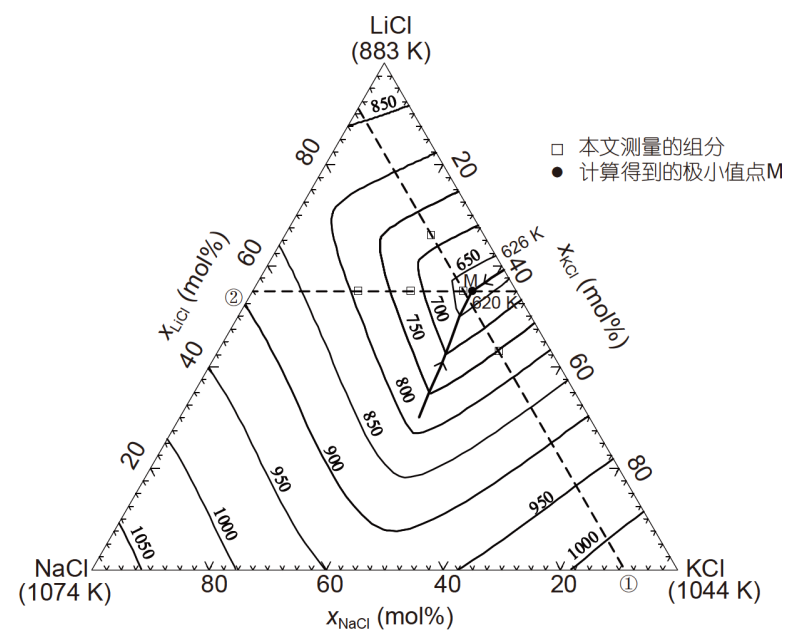

图 8 三元系 $\mathrm{LiCl}-\mathrm{NaCl}-\mathrm{KCl}$ 的液相面投影图

Figure 8 The liquidus projection of $\mathrm{LiCl}-\mathrm{NaCl}-\mathrm{KCl}$

热力学优化, 得到如下结论:

(1) $\mathrm{LiCl}-\mathrm{NaCl}$ 是二元匀晶体系，极小值点位于 $x_{\mathrm{NaCl}}=0.262$, 温度为 $820 \mathrm{~K}$. 与前人的优化结果相比, 本 文计算得到的固相线更接近实验值. 计算得到的该二 元系固溶体发生相分离的临界点温度在实验测量值范 围内. 因此, 本文得到的该体系模型参数更加合理.

(2) LiCl-NaCl-KCl的液相面有一个极小值点, 而不 是四相共晶点，对应的组分为 $55 \% \mathrm{LiCl}-8 \% \mathrm{NaCl}-37 \%$ $\mathrm{KCl}$, 温度为 $620 \mathrm{~K}$ ，与文献[4]中的共晶点实验值吻合 很好.

\section{参考文献}

1 Sangster J, Pelton A D. Phase diagrams and thermodynamic properties of the 70 binary alkali halide systems having common ions. J Phys Chem Ref Data, 1987, 16: 509-561

2 Tian S J, Liu S X, Zheng C G. Investigation on the phase diagram of the ternary system $\mathrm{LaCl}_{3}$-NaCl-LiCl. J Alloys Compd, 1998, 279: 127-131

3 Chartrand P, Pelton A D. Thermodynamic evaluation and optimization of the $\mathrm{LiCl}-\mathrm{NaCl}-\mathrm{KCl}-\mathrm{RbCl}-\mathrm{CsCl}-\mathrm{MgCl}_{2}-\mathrm{CaCl}_{2}$ system using the modified quasi-chemical model. Metall Mater Trans A, 2001, 32A: 1361-1383

4 Sangster J, Pelton A D. Thermodynamic calculation of phase diagrams of the 60 common-ion ternary systems containing cations Li, Na, K, Rb, Cs and anions $\mathrm{F}, \mathrm{Cl}$, Br, I. J Phase Equilib, 1991, 12: 511-537

5 Zhao J C. Methods for Phase Diagram Determination. Amsterdam: Elsevier, 2007

6 Barin I, Knacke O, Kubaschewski O. Thermochemical Properties of Inorganic Substances: Supplement. New York: Springer-Verlag, 1977

7 Pelton A D, Degterov S A, Eriksson G, et al. The modified quasichemical model I—Binary solutions. Metall Materi Trans B, 2000, 31: 651-659

8 Pelton A D, Chartrand P. The modified quasi-chemical model: Part II. Multicomponent solutions. Metall Mat Trans A, 2001, 32: 1355-1360

9 Hillert M. The compound energy formalism. J Alloys Compd, 2001, 320: 161-176

10 Barry T I, Dinsdale A T, Gisby J A, et al. The compound energy model for ionic solutions with applications to solid oxides. J Phase Equilib, 1992, 13: $459-475$

11 Klochko M. Double decomposition in the absence of a solvent. XXIV. Irreversible-reciprocal system: Sodium chloride-lithium sulfate. Zh Obshch Khim, 1933, 3: 1026-1039

12 Gromakov S, Gromakova L. Several rules for the plotting of phase diagrams of binary systems. Zh Fizich Khim, 1953, 27: 1545-1555

13 Hersh L S, Kleppa O J. Enthalpies of mixing in some binary liquid halide mixtures. J Chem Phys, 1965, 42: 1309-1322 


\title{
Experimental measurement and thermodynamic optimization of the phase diagram of $\mathrm{LiCl}-\mathrm{NaCl}-\mathrm{KCl}$ system
}

\author{
Guangxuan $\mathrm{Lu}^{1,2}$, Tianwang $\mathrm{Lai}^{1}$, Maogang $\mathrm{He}^{1 *} \&$ Xiangyang Liu ${ }^{1}$ \\ ${ }^{1}$ MOE Key Laboratory of Thermo-Fluid Science and Engineering, Xi'an Jiaotong University, Xi'an 710049, China; \\ ${ }^{2}$ Western Metal Materials Co., Ltd, Xi'an 710201, China \\ * Corresponding author, E-mail: mghe@mail.xjtu.edu.cn
}

Molten salt electrolysis is one of the most important methods to produce rare earth metals and their alloys. In this method, mixtures consisting of rare earth chlorides and alkali chlorides are usually used as electrolytes. The knowledge of the thermodynamic properties of electrolytes is essential to design and improve the electrolytic process. The relationship between the composition and the primary crystal temperature can be obtained through the phase diagram of the molten salt. Then the electrolysis temperature and electrolyte composition can be initially determined, which lays a foundation for further study of other properties. Since the most commonly used alkali chlorides in industry are $\mathrm{LiCl}, \mathrm{NaCl}$ and $\mathrm{KCl}$, the phase equilibrium data of $\mathrm{LiCl}-\mathrm{NaCl}-\mathrm{KCl}$ are bases for constructing the thermodynamic database of multicomponent chloride melts containing rare earths. This paper mainly focuses on the thermodynamic properties of the binary system $\mathrm{LiCl}-\mathrm{NaCl}$ and the ternary system LiCl-NaCl-KCl.

With regard to the binary system $\mathrm{LiCl}-\mathrm{NaCl}$, numerous researchers have used the thermal analysis method to experimentally measure its phase diagram. However, the data obtained by different researchers are quite different. Whether there exist intermediate compounds and whether it is an isomorphous phase diagram is still not confirmed. Furthermore, the existing experimental data were mostly measured by the cooling curve method from 1910 to 1960 and they are generally not consistent with each other. Besides, the deviation between the recently calculated phase diagram and experimental data is somewhat large. For instance, the temperature deviation between the calculated and measured solidus can be up to $30 \mathrm{~K}$. With respect to the ternary system $\mathrm{LiCl}-\mathrm{NaCl}-\mathrm{KCl}$, the measurement of the phase diagram has been performed by many researchers and the thermodynamic optimization has also been carried out. However, there is significant difference among these experimental data. And the results of previous optimization are inconsistent with FTsalt database of FactSage software. For example, there is an eutectic point in the liquidus projection of the phase diagram of $\mathrm{LiCl}-\mathrm{NaCl}-\mathrm{KCl}$ at $619 \mathrm{~K}$, while according to the FactSage software there is only a minimum instead of an eutectic point and the temperature is $621 \mathrm{~K}$. Therefore, the phase diagrams of $\mathrm{LiCl}-\mathrm{NaCl}$ and $\mathrm{LiCl}-\mathrm{NaCl}-\mathrm{KCl}$ systems were measured using differential thermal analysis in this work. Then these systems were thermodynamically optimized based on the measured data, including phase diagram, enthalpy of mixing, activity, etc, in previous and current work using CALPHAD method. During the optimization, the modified quasichemical model was used to describe the liquid solution while the Compound Energy Formulism was employed to model the solid solution.

According to our calculation, $\mathrm{LiCl}-\mathrm{NaCl}$ has an isomorphous phase diagram with a minimum at $x_{\mathrm{NaCl}}=0.262$ and $T$ $=820 \mathrm{~K}$. Compared with the previous optimization results, the solidus calculated in this paper is closer to the experimental value. The calculated temperature at which the solid solution undergoes phase separation is within the range of experimental data. Apart from that, there is a minimum in the liquidus projection of $\mathrm{LiCl}-\mathrm{NaCl}-\mathrm{KCl}$ system, rather than a four-phase eutectic point. The corresponding component is $55 \% \mathrm{LiCl}-8 \% \mathrm{NaCl}-37 \% \mathrm{KCl}$ and the temperature is $620 \mathrm{~K}$, which is in common with the literature. Therefore, the thermodynamic parameters obtained in this work are more reasonable.

LiCl-NaCl-KCl, modified quasichemical model, experimental measurement, thermodynamic optimization doi: 10.1360/TB-2019-0416 